\title{
¿Qué tan silenciosa es la hipoxemia en COVID-19?
}

\section{How silent is hypoxemia in COVID-19?}

\author{
José Rogelio Pérez-Padilla,* Ireri Isadora Thirión-Romero,* \\ Teresa Aguirre-Pérez,* Sebastián Rodríguez-Llamazares*
}

*Instituto Nacional de Enfermedades Respiratorias Ismael Cosío Villegas, Ciudad de México.

Dentro de la gama de presentaciones del COVID-19 se ha mencionado en algunas publicaciones la llamada «hipoxemia silenciosa».,2 Aunque la descripción no es uniforme, en general se refieren a pacientes que se sienten confortables y no se quejan de dificultad respiratoria, pero se ven cianóticos y se documenta hipoxemia. O bien, se documenta hipoxemia grave o muy grave en personas que a la exploración no se ven disneicas o con dificultad respiratoria, o que acudieron por diversas molestias que no incluían la dificultad para respirar y en los cuales se encontró neumonía con hipoxemia.

Básicamente se describe una desproporción entre la hipoxemia medida objetivamente y la dificultad para respirar subjetiva y objetiva.

Por un lado, estas observaciones resaltan la necesidad de las mediciones objetivas de hipoxemia, ya sea el oxímetro de pulso, o bien, la gasometría arterial ya que la observación de cianosis es poco confiable y depende de la cantidad absoluta de hemoglobina no oxigenada y del color de la piel.

Los estímulos para ventilar y para la taquipnea en un paciente con neumonía vienen de varias fuentes y la hipoxemia sólo es una, y no muy consistente o poderosa. La respuesta ventilatoria a la hipoxemia es muy variable, y hay un porcentaje de la población que prácticamente no incrementa su ventilación cuando va bajando su oxigenación en una prueba de respuesta a la hipoxemia aguda por rerrespiración. ${ }^{3}$ Estas personas con nula hiperventilación, a pesar de hipoxia grave, también suelen carecer de disnea.

Correspondencia:

Dr. José Rogelio Pérez Padilla

Instituto Nacional de Enfermedades Respiratorias

Ismael Cosío Villegas, Ciudad de México.

Correo electrónico: perezpad@gmail.com

Trabajo recibido: 24-V-2020; aceptado: 26-V-2020.
Esta respuesta tiene un componente genético pero pudiera ser más frecuente en residentes a alturas considerables y expuestos a hipoxemia prolongada. Las personas sin respuesta ventilatoria a la hipoxemia se considerarán de mayor riesgo en caso de una infección por COVID-19, ya que desarrollarían hipoxemia más rápidamente por la combinación de altitud y enfermedad pulmonar, y además no mostrarían una mayor ventilación ni una percepción adecuada de la hipoxemia. Más recientemente se ha atribuido al SARS-CoV-2 una afinidad sobre el tejido nervioso que podría contribuir a una baja percepción de la disnea. ${ }^{4}$

En contraste, es prácticamente universal que ante una re-respiración de $\mathrm{CO}_{2}$ se incremente la ventilación y la sensación de dificultad para respirar.

Otro estímulo para ventilar y para taquipnea procede de la fiebre y de receptores pulmonares tanto a estiramiento como a estímulos químicos que viajan por el nervio vago. ${ }^{5}$ Estos estímulos serían más leves en una fase temprana de edema pulmonar intersticial que en una fase de consolidación y por lo tanto generan menos molestias.

Es indispensable identificar y tratar la hipoxemia, que se asocia a un incremento en la mortalidad por COVID-19 ${ }^{6}$ así como por otras neumonías, y probablemente explica la mayor letalidad y hospitalización de la influenza con la altitud. ${ }^{78}$ La vasoconstricción hipóxica reduce el flujo sanguíneo a zonas hipoxémicas del pulmón y el desbalance en la relación ventilación/perfusión en las neumonías, y parece ser un mecanismo con funcionalidad reducida en el COVID-19 y podría ser un blanco terapéutico adicional. Se ha demostrado en neumonías bacterianas en modelos animales que la vasoconstricción hipóxica se reduce o se pierde incrementándose en consecuencia el cortocircuito y la hipoxemia, y al menos en algunos modelos se recupera parcial o totalmente con indometacina o aspirina. . $^{9-11}$ Los productos de la inflamación pueden pues inhibir la vasoconstricción hipóxica y empeorar el cortocircuito o hipoxemia en COVID-19,12 pero de manera interesante 
parece ocurrir en forma temprana desde la fase de vidrio despulido con edema intersticial, y no sólo en la fase de consolidación. Queda por verse si el uso de algún antiinflamatorio pudiera compensar la hipoxemia a través de mejorar la vasoconstricción hipóxica, ya que en un estudio de neumonía en humanos el resultado fue heterogéneo, aunque sí se encontró un grupo con mejoría importante en la oxigenación. ${ }^{9}$

Varios lugares de México situados a una altura considerable sobre el nivel del mar se consideran de alto riesgo para COVID-19 porque además combinan condiciones socioeconómicas desfavorables y exposición amplia al humo de leña. ${ }^{13}$

En resumen, la identificación de la hipoxemia se debe hacer por un oxímetro de pulso o una gasometría arterial y no quedará oculta ni silenciosa. La falta o escasez de disnea se esperaría sobre todo en sujetos con pobre respuesta a la hipoxemia y sin condensaciones en la tomografía. Es indispensable contar con oximetría de pulso, especialmente en comunidades localizadas a alturas significativas, para identificar tempranamente hipoxemia y además tener acceso a oxígeno suplementario, ambas medidas son accesibles y pueden salvar vidas especialmente durante la pandemia de COVID-19.

\section{REFERENCIAS}

1. Xie J, Tong Z, Guan X, Du B, Qiu H, Slutsky AS. Critical care crisis and some recommendations during the COVID-19 epidemic in China. Intensive Care Med 2020;46(5):837-840. https://doi.org/10.1007/ s00134-020-05979-7

2. Ottestad W, Seim M, Maehlen JO. COVID-19 with silent hypoxemia. Tidsskr Nor Laegeforen 2020;140(7): https://doi.org/10.4045/ tidsskr.20.0299

3. Vazquez-Garcia JC, Arellano-Vega SL, Regalado-Pineda J, PerezPadilla JR. Normal ventilatory response to hypoxia and hypercapnia at an altitude of 2240 meters. Rev Invest Clin. 1998;50(4):323-329.
4. Coen M, Allali G, Adler D, Serratrice J. Hypoxemia in COVID-19; Comment on: "The neuroinvasive potential of SARS-CoV2 may play a role in the respiratory failure of COVID-19 patients". J Med Virol 2020;10. https://doi.org/10.1002/jmv.26020

5. LeeLY. Respiratorysensations evoked by activation of bronchopulmonary C-fibers. Respir Physiol Neurobiol 2009;167(1):26-35. https://doi. org/10.1016/j.resp.2008.05.006

6. Xie J, Covassin N, Fan Z, et al. Association between hypoxemia and mortality in patients with COVID-19. Mayo Clinic proceedings 2020;95(6):P1138-1147. https://doi.org/10.1016/j.mayocp.2020.04.006

7. Pérez-Padilla R, García-Sancho C, Fernández R, Franco-Marina F, López-Gatell H, Bojórquez I. The impact of altitude on hospitalization and hospital mortality from pandemic 2009 influenza $A$ (H1N1) virus pneumonia in Mexico. Salud Publica Mex 2013;55(1):92-95. https:// doi.org/10.1590/s0036-36342013000100013

8. Pérez-Padilla R, Franco-Marina F. The impact of altitude on mortality from tuberculosis and pneumonia. Int J Tuberc Lung Dis 2004;8(11):1315-1320.

9. Hanly PJ, Roberts D, Dobson K, Light RB. Effect of indomethacin on arterial oxygenation in critically ill patients with severe bacterial pneumonia. Lancet 1987;1(8529):351-354. https://doi.org/10.1016/ s0140-6736(87)91727-2

10. Light RB. Indomethacin and acetylsalicylic acid reduce intrapulmonary shunt in experimental pneumococcal pneumonia. Am Rev Respir Dis 1986;134(3):520-525. https://doi.org/10.1164/arrd.1986.134.3.520

11. McCormack DG, Crawley DE, Evans TW. New perspectives in the pulmonary circulation and hypoxic pulmonary vasoconstriction. Pulm Pharmacol 1993;6(2):97-108. https://doi.org/10.1006/ pulp.1993.1013

12. Lang M, Som A, Mendoza DP, et al. Hypoxaemia related to COVID-19: vascular and perfusion abnormalities on dual-energy CT. Lancet Infect Dis 2020. https://doi.org/10.1016/s1473-3099(20)30367-4

13. Masera O, Riojas-Rodríguez $\mathrm{H}$, Pérez-Padilla $\mathrm{R}$, et al. Vulnerabilidad a COVID-19 en poblaciones rurales y periurbanas por el uso doméstico de leña. 2020 [Available from: https://http:// www.insp.mx/avisos/5386-vulnerabilidad-covid-19-poblacionesrurales.html 